\title{
Response of Ventricular Repolarization to Simulated Microgravity Measured by Periodic Repolarization Dynamics Using Phase-Rectified Signal Averaging
}

\author{
Saúl Palacios ${ }^{1}$, Enrico Gianluca Caiani ${ }^{2}$, Esther Pueyo ${ }^{1,3}$, Juan Pablo Martínez ${ }^{1,3}$ \\ ${ }^{1}$ BSICoS, Aragon Institute of Engineering Research, IIS Aragón, Universidad de Zaragoza, \\ Zaragoza, Spain \\ ${ }^{2}$ Dipartimento di Elettronica, Informazione e Bioingegneria, Politecnico di Milano, Milano, Italy \\ ${ }^{3}$ CIBER en Bioingeniería, Biomateriales y Nanomedicina (CIBER-BBN), Spain
}

\begin{abstract}
Head-Down Bed Rest (HDBR) microgravity simulation induces cardiovascular deconditioning, including effects on ventricular repolarization. The index of Periodic Repolarization Dynamics (PRD) was developed to quantify low-frequency oscillations of cardiac repolarization. In this study, PRD was quantified by Phase Rectified Signal Averaging $\left(P R D_{P R S A}\right)$ and Continuous Wavelet Transform $\left(P R D_{C W T}\right)$ methods. PRD was analyzed in ECGs from 22 volunteers at rest and during orthostatic Tilt-Table Test (TTT) performed before and after $-6^{\circ}$ 60-days HDBR. Significant correlation was found between PRD measured by PRSA and CWT (Pearson's $\rho=0.93, p<10^{-54}$ and Kendall's $\tau=0.79 p<10^{-38}$ ). A highly significant increase was found when $P R D_{P R S A}$ values were measured at POST-HDBR with respect to PRE-HDBR in the tilt phase: 1.40 [1.10] deg and 0.97 [0.90] deg (median [IQR]), $p=0.008$, respectively. $P R D_{P R S A}$ also increased significantly in the tilt phase with respect to baseline, both at POST-HDBR (0.90 [0.57] deg, $p=0.003)$ and at PRE-HDBR (0.75 [0.45] deg, $p=0.011)$. PRD, either measured with PRSA or with CWT, is able to measure changes in ventricular repolarization induced by microgravity simulation as well as following sympathetic provocation.
\end{abstract}

\section{Introduction}

Human space exploration has a number of associated risks and challenges. Changes in the physical forces on and within the body brought about by microgravity exposure represent a real risk to the astronauts' health [1]. Weightlessness effects on the human body include muscular, cardiac and bone-related [2]. Orthostatic intolerance is a known relevant cardiovascular issue experienced by astronauts after landing. It is due to cardiac deconditioning, with some astronauts not even being able to remain stand- ing upright after prolonged stay in orbit. Both the sympathetic and parasympathetic branches of the autonomic nervous system are involved in orthostatic regulation [3].

The orthostatic Tilt-Table Test (TTT) is a procedure commonly used in the management of reflex syncope, due to cardiac autonomic reflex [4], and is used to assess autonomic response. Head-down bed rest (HDBR) is a reliable ground-based model that simulates most effects of spaceflight on the human body [5]. Few studies have investigated microgravity effects on the heart's electrical activity, with major findings indicating prolonged cardiac conduction and repolarization duration [6] in association with long duration spaceflight. In this work, ECG signals were recorded under an HDBR model and analyzed to evaluate microgravity effects on ventricular repolarization.

Periodic Repolarization Dynamics (PRD) is a recently proposed index to assess sympathetically-modulated ventricular repolarization by measuring low-frequency $(\leq 0.1$ $\mathrm{Hz}$ ) oscillations in the T-wave angle of the body-surface electrocardiogram (ECG) [7]. The Continuous Wavelet Transform has been proposed as a method to quantify PRD $\left(P R D_{C W T}\right)$ [7]. Phase-Rectified Signal Averaging [8] has also been proposed as a simpler alternative $\left(P R D_{P R S A}\right)$.

The aim of this study is to compare both methods for PRD quantification $\left(P R D_{C W T}\right.$ and $\left.P R D_{P R S A}\right)$ and to assess the ability of $P R D_{P R S A}$ to measure autonomic changes induced by TTT-based sympathetic provocation and changes induced by prolonged bed rest.

\section{Materials}

Twenty-two healthy male volunteers $(29 \pm 6$ years, $181 \pm 5 \mathrm{~cm}, 77 \pm 7 \mathrm{~kg}$ ) participated in a 60 -day $-6^{\circ} \mathrm{HDBR}$ experiment. They were randomly divided into countermeasure group (JUMP), who exercised on a sledge jump system [9] during the HDBR time period, and control group (CTRL), not performing any exercise. This experiment took place in the :envihab facility of the Institute 
of Aerospace Medicine, German Aerospace Center-DLR (Cologne, Germany). It is part of the European Space Agency (ESA) bed rest studies.

The whole campaign consisted of three phases (Figure 1): 15 days of pre-bed rest (BDC-15 to BDC-1), 60 days of HDBR (HDT1 to HDT60) and 15 days of post-bed rest recovery $(\mathrm{R}+0$ to $\mathrm{R}+14)$. Each subject participated in a TTT at pre- (2 days before HDBR, BCD-2) and post-HDBR (just after completing HDBR, $\mathrm{R}+0$ ) to assess autonomic response. The maximal duration of each TTT was 15 minutes with the subject tilted head-up to an angle of $80^{\circ}$. After this 15 minutes, a step rising $(3 \mathrm{~min})-10 \mathrm{mmHg}$ was applied through a Lower Body Negative Pressure (LBNP) chamber in which the subject was inside up to the abdomen.

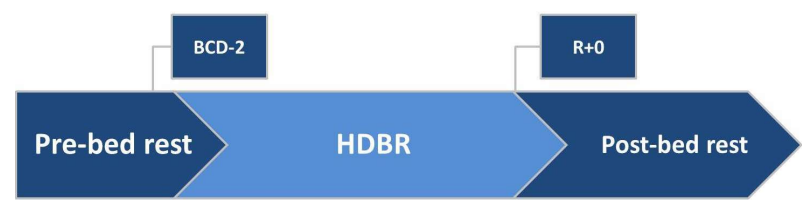

Figure 1. Phases of the long-term microgravity campaign, with indication of the days when volunteers underwent TTT.

12-lead ECG signals (Mortara Instrument, Inc., Milwaukee, WI, USA, $1000 \mathrm{~Hz}$ sampling frequency) were available for this study. 5-minute segments from the control phase prior to TTT, from the beginning of tilt phase and from the end of the tilt phase (after LBNP application, when it was applied) were analyzed. If the tilt phase duration was less than 5 minutes, which happened in few cases, the whole tilt interval was analyzed.

All subjects underwent a prior comprehensive medical examination during the selection process, were fully informed of the experiment and provided written, informed consent to participate in the study, which was approved by the Ethical Committee for Human Research at the host institution.

\section{Methods}

\subsection{Preprocessing}

Preprocessing of ECG recordings included noise removal using a $50 \mathrm{~Hz}$ notch filter and a $40 \mathrm{~Hz}$ Finite Impulse Response filter. Baseline wander was cancelled by cubic splines interpolation.

\subsection{Series of angles between consecutive $T$ - wave angles}

For PRD quantification, the series of angles between consecutive $T$ waves were obtained based on [7], as de- scribed in the following:

1. Orthogonal leads X, Y, Z were obtained from each 12lead ECG recording by using the inverse Dower matrix.

2. T-wave onsets $\left(\mathrm{T}_{o n}\right)$ and ends $\left(\mathrm{T}_{o f f}\right)$ were identified using a wavelet-based automatic system [10]. For unidentified T-wave onsets, $\mathrm{T}_{\text {on }}$ was calculated based on $\mathrm{T}_{\text {on }}$ temporal locations in adjacent beats. Specifically, $\mathrm{T}_{o n}$ was located at a distance from the QRS fiducial point equal to the median time duration between the QRS and $\mathrm{T}_{\text {on }}$ marks in 30 beats around it.

3. To get T-wave segments of the same duration for the comparison of two T-waves, while ensuring that all samples are within the $\mathrm{T}$-wave, both $\mathrm{T}_{o n}$ and $\mathrm{T}_{o f f}$ were redefined according to the following criteria:

- $T_{o n}$. Comparing the time difference between QRS fiducial point and $\mathrm{T}_{\text {on }}$ for two consecutive beats, the largest value was chosen.

- $T_{o f f}$. Comparing the time difference between QRS and $\mathrm{T}_{\text {off }}$ for two consecutive beats, the smallest value was chosen.

4. The reference point was set the end of the segment for each T-wave. The angle $\mathrm{dT}^{\circ}$ between two consecutive $\mathrm{T}$ wave average vectors was computed using the dot product, considering the segments previously described. $\mathrm{dT}^{\circ}$ represents the angular change between two successive repolarizations.

5. A 10th-order median filter was applied to the series of $\mathrm{dT}^{\circ}$ values to attenuate abrupt variations.

At this point, two different methods were applied onto the $\mathrm{dT}^{\circ}$ time series to calculate the PRD index: Continuous Wavelet Transform $\left(P R D_{C W T}\right)$ and Phase-Rectified Signal Averaging $\left(P R D_{P R S A}\right)$.

\subsection{PRD using Continuous Wavelet Trans- form}

Once the beat-to-beat $\mathrm{dT}^{\circ}$ series was obtained, the Continuous Wavelet Transform (CWT) was applied following the next steps [7]:

1. A uniformly sampled signal at $2-\mathrm{Hz}$ was obtained by linear interpolation and a 30-sample moving average was applied to remove artifacts.

2. Wavelet coefficients were obtained by applying a 4thorder Gaussian wavelet for each scale at each time point. An average wavelet coefficient was computed for each scale.

3. Scales $(a)$ were converted to pseudo-frequencies $\left(F_{a}\right)$ according to the following equation:

$$
F_{a}=\frac{F_{c}}{a \cdot \Delta}
$$

where $F_{c}$ is the center frequency of the mother wavelet and $\Delta$ denotes the sampling period. 
$P R D_{C W T}$ was defined as the average wavelet coefficient in the frequency range between 0.025 and $0.1 \mathrm{~Hz}$.

\subsection{PRD using Phase-Rectified Signal Av- eraging}

PRD index using Phase-Rectified Signal Averaging (PRSA) method was obtained by applying the following steps [8]:

1. Anchor points in the $\mathrm{dT}^{\circ}$ series were defined as the points $x_{i}$ where the average of their $T$ prior values was greater than the average of their $T$ subsequent values, using $T=9$ :

$$
\frac{1}{T} \sum_{j=0}^{T-1} x_{i+j}>\frac{1}{T} \sum_{j=1}^{T} x_{i-j}
$$

2. Windows of $L=40$ values were defined around each anchor point. Anchor points in the first or the last $L / 2=$ 20 beats of the series were discarded.

3. PRSA signal was obtained by averaging over all windows.

$P R D_{P R S A}$ was defined as the maximum peak-to-peak amplitude of PRSA signal.

\subsection{Statistical analysis}

Wilcoxon signed-rank test was used for comparison of paired samples (pre-HDBR vs post-HDBR or between TTT phases). Spearman's correlation $(\rho)$ and Kendall's $\tau$ were used to quantify rank correlation between CWT and PRSA. For all tests, the null hypothesis was rejected if $p \leq 0.05$.

\section{Results and Discussion}

\subsection{Comparison of PRD measured with CWT and PRSA}

Figure 2 shows PRD values quantified by CWT (X-axis) and PRSA (Y-axis) for all analyzed segments (from all subjects, TTT phases and HDBR stages). Results show a very significant positive rank correlation: Spearman's $\rho=0.93\left(p<10^{-54}\right)$; Kendall's $\tau=0.79\left(p<10^{-38}\right)$. These coefficients indicate a strong concordance between both indices, as previously reported in [7].

\subsection{Effect on PRD index due to tilt test and microgravity}

Each subject underwent TTT before (PRE) and after (POST) HDBR. For each TTT, baseline (prior to the tilt phase) as well as beginning and end of the tilt phase were analyzed. This test induced changes in cardiac autonomic

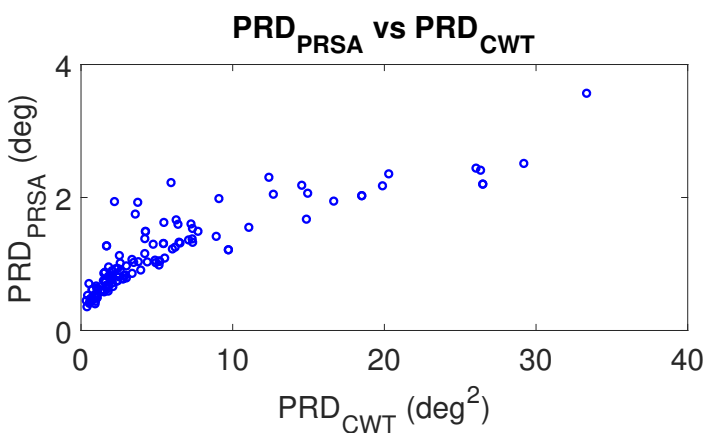

Figure 2. Scatter plot of PRD values measured by CWT and PRSA algorithm. Data are shown together regardless of the TTT phase or whether they correspond to pre- or post-HDBR.

modulation that both $P R D_{C W T}$ and $P R D_{P R S A}$ were able to measure. The main results obtained by $P R D_{C W T}$ are available in [11]. Figure 3 shows general increases in PRD values both at PRE and at POST. Differences are more marked when evaluated with $P R D_{P R S A}$ than with $P R D_{C W T}$. This increase supports the fact that PRD, evaluated by both methods, is enhanced in response to sympathetic activation [7]. This is in agreement with previous studies showing that low-frequency oscillations of ventricular repolarization duration are magnified following sympathetic provocation $[12,13]$. Potential mechanisms underlying such oscillations have been postulated $[12,13]$.

At each phase, i.e. baseline, beginning and end of tilt, the effect of microgravity simulation on ventricular repolarization was evaluated. Figure 3 shows PRD differences between PRE and POST HDBR measured by $P R D_{C W T}$ (upper panel) as well as by $P R D_{P R S A}$ (lower panel). At baseline, $P R D_{P R S A}$ was higher at POST with respect to PRE. Futhermore, for both beginning and end of tilt, PRD was significantly enhanced at POST with respect to PRE, regardless of the quantification method. The observed increase was even more clear at the beginning of tilt, where $P R D_{P R S A}$ changed from 0.97 [0.90] deg at PRE to 1.40 [1.10] deg at POST. At the end of tilt, PRD increased from 0.95 [0.82] deg at PRE to 1.27 [0.96] deg at POST. All evaluated PRD values, both $P R D_{C W T}$ and $P R D_{P R S A}$, are presented in Table 1.

These results reinforce the idea that relevant alterations in ventricular repolarization occur when subjects are exposed to a long-term microgravity environment $[14,15]$. Such alterations become even more manifested following stimulation of the sympathetic nervous system.

\section{Conclusion}

PRD values calculated by CWT and PRSA techniques are equivalent in terms of rank statistics. For both meth- 

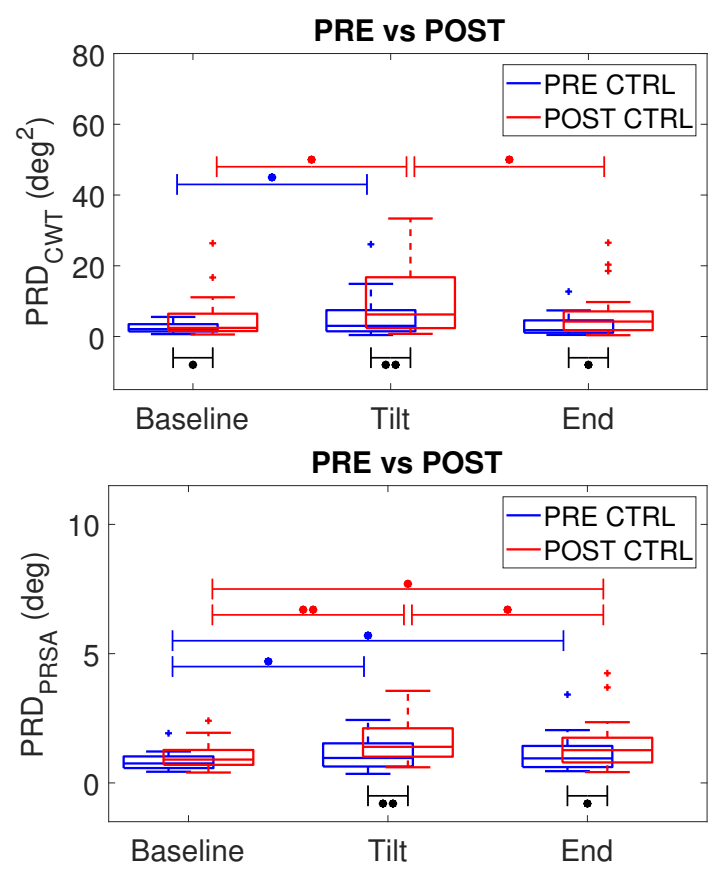

Figure 3. Boxplots of $P R D_{C W T}$ (upper panel) and $P R D_{P R S A}$ (lower panel) values, evaluated at baseline and during the beginning and end of TTT. $* * p<0.01,{ }^{*} \mathrm{p}<0.05$

\begin{tabular}{|c|c|c|c|}
\hline & $\begin{array}{c}\text { PRSA } \\
(\text { deg })\end{array}$ & $\begin{array}{c}\text { CWT } \\
\left(\operatorname{deg}^{2}\right)\end{array}$ \\
\hline & & Median [IQR] & Median [IQR] \\
\hline \multirow{2}{*}{ Baseline } & PRE & $0.75[0.45]^{\dagger}$ & $2.10[2.06]^{\dagger}$ \\
\hline & POST & $0.90[0.57]^{\dagger \dagger}$ & $2.45[4.91]^{* \dagger}$ \\
\hline \multirow{2}{*}{ Tilt } & PRE & $0.97[0.90]$ & $3.02[5.98]$ \\
\hline & POST & $1.40[1.10]^{* *}$ & $6.24[14.38]^{* *}$ \\
\hline \multirow{2}{*}{ End } & PRE & $0.95[0.82]^{\times}$ & 1.79 [3.50] \\
\hline & POST & $1.27[0.96]^{* \dagger \times}$ & $4.23[5.31]^{* \dagger}$ \\
\hline
\end{tabular}

Table 1. PRD values at all phases of TTT for PRE and POST. ${ }^{* *} \mathrm{p}<0.01,{ }^{*} \mathrm{p}<0.05$ (respect to PRE), ${ }^{\dagger \dagger} \mathrm{p}<0.01$, ${ }^{\dagger} \mathrm{p}<0.05$ (respect to tilt), ${ }^{\times} \mathrm{p}<0.05$ (respect to baseline)

ods, the major findings of this work reveal that simulated microgravity induces alterations in autonomic modulation of ventricular repolarization and that TTT leads to notable increases in PRD both before and after exposure to simulated microgravity.

\section{Acknowledgements}

This work was supported by projects ERC-2014StG 638284 (ERC), DPI2016-75458-R (MINECO) and Aragón Government (Reference Group BSICoS T39-17R) cofunded by FEDER 2014-2020. We also acknowledge the support of the Italian Space Agency (contract 2018-7-U.0, $\mathrm{PI}$ and recipient EG Caiani).

\section{References}

[1] White RJ, Averner M. Humans in space. Nature. 2001; 409:1115-1118.

[2] Antonutto G, Di Prampero PE. Cardiovascular deconditioning in microgravity: some possible countermeasures. Eur J Appl Physiol. 2003; 90:283-91.

[3] Stewart JM. Mechanisms of sympathetic regulation in orthostatic intolerance. J Appl Physiol. 2012; 113:1659-1668.

[4] Teodorovich N, Swissa M. Tilt table test today - state of the art. World J Cardiol 2016; 8:277282.

[5] Jost PD. Simulating human space physiology with bed rest. Hippokratia 2008; 12(1): 37-40.

[6] D'Aunno DS et al. Effect of short-and long-duration spaceflight on QTc intervals in healthy astronauts. Am J Cardiol. 2003; 91(4):494-7.

[7] Rizas KD et al. Periodic repolarisation dynamics: A natural probe of the ventricular response to sympathetic activation. Arrhythm Electrophysiol Rev. 2016; 5(1):3136.

[8] Bauer A et al. Phase-rectified signal averaging detects quasi-periodicities in non-stationary data. Physica A. 2006; 364:423-434

[9] Kramer A et al. High-intensity jump training is tolerated during 60 days of bed rest and is very effective in preserving leg power and lean body mass: An overview of the Cologne RSL study. PLOS ONE. 2017; 12(1):e0169793

[10] Martínez JP et al. A wavelet-based ECG delineator: Evaluation on standard databases. IEEE Trans Biomed Eng. 2004; 51(4):570-581.

[11] Palacios S, Caiani EG, Martínez JP, Pueyo E. Microgravity exposure alters sympathetic modulation of ventricular repolarization quantified from the ECG via periodic repolarization dynamics. In Computing in Cardiology 2018, vol 45. DOI: $10.22489 /$ CinC.2018.358

[12] Pueyo E, Orini M, Rodrguez JF, Taggart P. Interactive effect of beta-adrenergic stimulation and mechanical stretch on low-frequency oscillations of ventricular action potential duration in humans. J Mol Cell Cardiol. 2016; 97:93-105.

[13] Porter B, Bishop MJ, Claridge S, Behar J et al. Autonomic modulation in patients with heart failure increases beatto-beat variability of ventricular action potential duration. Front Physiol. 2017; 8:328.

[14] Bolea J, Caiani EG, Pueyo E, Laguna P, Almeida R. Microgravity effects on ventricular response to heart rate changes, Proc. Ann. Int. Conf. IEEE Eng. Med. Biol. Soc., EMBS 2012; 3424-3427

[15] Caiani EG, Pellegrini A, Bolea J, Sotaquira M, Almeida R, Vaïda P. Impaired T-wave amplitude adaptation to heartrate induced by cardiac deconditioning after 5-days of headdown bed-rest. Acta Astronaut 2013; 91:166-172.

Address for correspondence:

Saúl Palacios

Lab 3.07, Edificio Ada Byron

María Luna 1, 50018 Zaragoza (Spain)

spalacios@unizar.es 